\title{
Comparison of the Efficacy and Tolerability of Dienogest and Dienogest Plus Ethinylestradiol on Endometriosis Related-Pain
}

\author{
Tolga KARACAN'1, Huseyin KIYAK², Eser OZYUREK¹, Mevlide SAN', Engin ORAL ${ }^{3}$ \\ Istanbul, Turkey
}

\begin{abstract}
OBJECTIVE: Endometriosis is a common gynecological condition that affects many women of reproductive age worldwide and is a major cause of pain and infertility. Treatment of endometriosis can be either surgical, aiming to restore normal anatomy by removing endometriotic lesions, or hormonal. Various medical treatments with different doses, formulations, delivery systems, and regimens have been tested. The main objective of this study was to compare the efficacy and tolerability of dienogest and dienogest plus ethinylestradiol on endometriosis-related pain. Additionally, the effects on endometrioma size were examined.
\end{abstract}

STUDY DESIGN: A total of 81 patients with clinically diagnosed endometrioma, who had chronic pelvic pain, dysmenorrhea, or deep dyspareunia between January 2015 and December 2018 were studied retrospectively. The patients were divided into two main groups: continuous oral dienogest $(n=43)$ (Visanne $^{\circledR}, 2 \mathrm{mg} /$ day) and continuous oral dienogest plus ethinylestradiol $(\mathrm{n}=38)$ (Dienille ${ }^{\circledR}, 2 \mathrm{mg} / 0.03$ $\mathrm{mg} / \mathrm{day}$ ). The intensity of pain symptoms was evaluated before therapy, then after 3 and 6 months of treatment using a 10 -point numerical rating scale $(0=$ no pain and $10=$ worst possible pain) (NRS) provided to the patients in advance.

RESULTS: The pain scores related to chronic pelvic pain decreased $36 \%$ for dienogest and $49 \%$ for dienogest plus ethinylestradiol $(p<0.05)$ and scores for dysmenorrhea decreased $38 \%$ and $44 \%$ respectively $(p<0.05)$ at 6 months, significantly lower than before treatment. At the 6 -month follow-up, a $28 \%$ decrease in the pain scores related to deep dyspareunia in the dienogest group was statistically significant. Although the dienogest plus ethinylestradiol group also decreased by $20 \%$, the difference was not significant. There was no significant difference in endometrioma size between the two groups at the 6-month follow-up (dienogest and dienogest plus ethinylestradiol; $24.2 \pm 17.5 \mathrm{~mm}$ vs. $27.5 \pm 19.1$ $\mathrm{mm}$, respectively; $p=0.42$ ).

CONCLUSION: Upon analysis of our 6 months of clinical data, estrogen-progestin and a progestin alone seem to be of similar efficacy for the temporary treatment of endometriosis-related pain. The dienogest plus ethinylestradiol combination was slightly less effective on deep dyspareunia but was still well tolerated. Similarly, the two hormonal regimens posed no superiority over one another with regard to endometrioma size reduction.

Keywords: Dienogest, Dienogest plus ethinylestradiol, Endometriosis, Hormonal treatment

Gynecol Obstet Reprod Med 2020;26(3):192-198

${ }^{1}$ Department of Obstetrics and Gynecology University of Health Sciences Bagcilar Research and Education Hospital, Istanbul, Turkey

${ }^{2}$ Department of Obstetrics and Gynecology, University of Health Sciences, Kanuni Sultan Suleyman Research and Education Hospital, Istanbul, Turkey

${ }^{3}$ Department of Obstetrics and Gynecology, Istanbul University Cerrahpasa Medical Faculty, Istanbul, Turkey

Address of Correspondence.

Tolga Karacan

Merkez Mah. Mimar Sinan Cad. 6. Sokak, 34100, 34200 Bagcilar, Istanbul, Turkey

tolgakaracan84@gmail.com

Submitted for Publication: Accepted for Publication: ORCID IDs of the authors: EO: 0000-0002-5373-3991, 31.03.2019

24.05.2019

TK: 0000-0002-3510-4147,

HK: $0000-0001-7580-9179$

EO: $0000-0002-4123-6357$

\begin{tabular}{|c|c|}
\hline Quick Response Code: & Access this article online \\
\cline { 2 - 2 } & $\begin{array}{l}\text { Website: www.gorm.com.tr } \\
\text { e- mail: info@gorm.com.tr }\end{array}$ \\
\cline { 2 - 3 } & DOI:10.21613/GORM.2019.944 \\
\hline
\end{tabular}

\section{Introduction}

Endometriosis is a common gynecological condition that affects many women of reproductive age worldwide and is a major cause of pain and infertility. Treatment of endometriosis can be either surgical or hormonal. Surgical treatment aims to restore normal anatomy by removing endometriotic lesions. Hormonal therapy aims to induce a hypo-estrogenic state, atrophy or quiescence of endometriotic lesions, and a reduction of the chronic peritoneal inflammatory state. In the last decade, substantial progress in diagnostic imaging has allowed a reliable noninvasive diagnosis of endometriosis (i.e., without the need for surgical and histologic confirmation),

How to cite this article: Karacan T. Kiyak H. Ozyurek E. San M. Oral E. Comparison of the Efficacy and Tolerability of Dienogest and Dienogest Plus Ethinylestradiol on Endometriosis Related-Pain. Gynecol Obstet Reprod Med 2020;26(3):192-198 
thus opening the possibility to shift first-line treatment of endometriosis from surgery to medical therapy (1).

Hormonal therapy is not a cure; treatments often need to be administered for years to prolong their effects. Various medical treatments with different doses, formulations, delivery systems, or regimens focusing on endometriosis treatment have been studied. According to the guidelines on endometriosis management issued by major international scientific societies, different medications such as oral contraceptives (OCs) and progestogens vary in safety and tolerability, despite having substantially similar effects in terms of pain relief. Consequently, progestogens and estroprogestins represent the first choice for the medical treatment of endometriosis (2-4).

Progestins are synthetic compounds that mimic the effects of progesterone. Small structural changes between them can account for considerable differences in their pharmacological properties and actions. With few exceptions, they are used to achieve similar results on target tissues, including suppression of ovarian function, decidualization, and atrophy of ectopic implants, and reduction of intraperitoneal inflammation. However, they are involved in different endocrine pathways owing to their chemical structure and pharmacological properties. In recent decades, newer progestins have been synthesized with the goal of finding a structure with no androgenic or glucocorticoid effects. These newer progestins, such as dienogest, in addition to having a strong pregestational action, also exert anti-estrogenic, anti-gonadotropic, and anti-mineralocorticoid effects. These compounds are included in combined oral contraceptives (5-7).

To the best of our knowledge, this is the first study to compare the effects of these two products (dienogest only vs. dienogest plus ethinylestradiol) on disease. The main objective of this study was to compare the efficacy and tolerability of dienogest and dienogest plus ethinylestradiol on endometriosis-related pain. In addition, the effects on endometrioma size were reported.

\section{Material and Method Data collection}

A total of 81 patients with clinically diagnosed endometrioma, who had chronic pelvic pain, dysmenorrhea, or deep dyspareunia between January 2015 and December 2018 were studied retrospectively. Subjects with complete data for the 6month period of hormonal treatment were included in the study. The study was approved by the local institutional review board (date: 03/02/2019 and ethics committee number: 2019.02.1.01.002.r1.015), and written informed consent was obtained from all participants. The strengthening the reporting of observational studies in epidemiology (STROBE) statement principles were applied for this retrospective cohort study. The patients were selected using the International Classification Database (ICD) codes from the electronic data- base at the hospital. Variables including age, medical treatment start date, body mass index (BMI) in $\mathrm{kg} / \mathrm{m}^{2}$, parity number, smoker status, infertility status, age at first menstruation, pain scores, previous endometriosis surgery, and endometrioma size were recorded.

\section{Design}

In this retrospective cohort analysis, the patients were divided into two main groups: continuous oral dienogest $(n=43)$ (Visanne ${ }^{\circledR}, 2 \mathrm{mg} /$ day) and continuous oral dienogest plus ethinylestradiol $(\mathrm{n}=38)$ (Dienille ${ }^{\circledR}, 2 \mathrm{mg} / 0.03 \mathrm{mg} /$ day) as shown in figure 1. Patients were informed that the provided medical treatment is not a cure for endometriosis and that pain symptoms could return when treatment was discontinued. After all the benefits and adverse effects were described, medications were given according to patient preference or the specialist's decision in patients who had no preference.

Criteria for inclusion in the study were: diagnosis of ovarian endometriosis based on ultrasonography (transvaginal/ transrectal) and supported by magnetic resonance imaging (MRI) if necessary, endometriosis-related pain symptoms, and desire to avoid surgery.

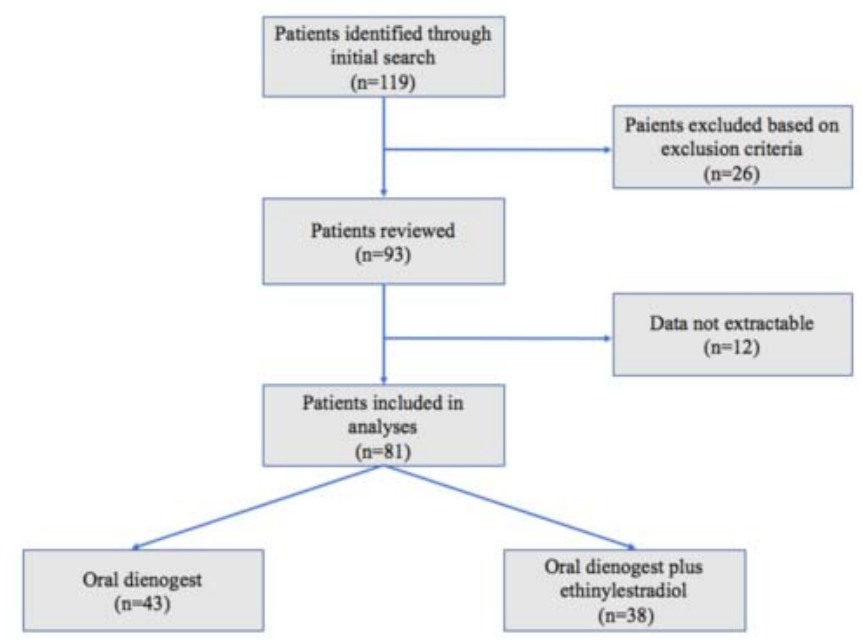

Figure 1: Flowchart of our retrospective study design

The exclusion criteria of the study were as follows: need for surgical treatment (suspicion of malignancy, obstructive uropathy, or bowel stenosis), therapies for endometriosis other than nonsteroidal anti-inflammatory drugs in the 6 months before inclusion in the study ( $\mathrm{GnRH}$ agonist, combined oral contraceptive), reluctance to tolerate menstrual changes, desire to conceive, the usual contraindications to estrogens and progestins, and concomitant pelvic inflammatory disease.

Standardized examination techniques, terms, and definitions were used. The preoperative classification of adnexal masses was determined in accordance with the recommendations of the International Ovarian Tumor Analysis (IOTA) group (8). Ovarian endometrioma was defined as a round- 
shaped unilocular or multilocular (less than five locules) cystic mass with regular margins, homogeneous low echogenic fluid content with scattered internal echoes, and without papillary projections. To rule out functional cysts, the presence of endometrioma was confirmed during at least two separate examinations performed at least one month apart to rule out other hemorrhagic cysts (9). The largest diameter of the endometrioma on sonographic view was recorded as the size of the ovarian endometrioma. If endometrioma was present in both ovaries, the total size was estimated by adding the largest diameters of the two endometriomas. No bowel preparation was performed prior to sonography.

The medical histories of the patients were taken verbally by the gynecologists during face-to-face appointments at the outpatient clinic. The first subjective impressions made during the physical examinations and transvaginal ultrasonography performed by the same gynecologists were recorded in the computer-based patient record system. Each scan was interpreted prospectively in real time. Pelvic pain symptoms evaluated in our study include dysmenorrhea (painful menstrual cramps), deep dyspareunia (pain with deep penetration during intercourse or sexual activity), and chronic pelvic pain (pain in the pelvic area that lasts for 6 months or longer). A numerical rating scale (NRS), with " 0 " indicating no pain and " 10 ", indicating the worst possible pain, was used (10). Women were asked to rate the current intensity of painful menstrual cramps, pain with deep penetration during intercourse or sexual activity, and pain in the pelvic area that had lasted for the preceding 6 months or longer. The diagnostic examination always included kidney and urinary tract ultrasonography.

Every 3 months, the patients underwent clinical assessment, vaginal and rectal examinations, transvaginal and transrectal ultrasonography, and were asked to complete the pain questionnaire again. In the absence of regular flows, pain during erratic bleeding episodes was considered as dysmenorrhea. Dysmenorrhea in women who were completely amenorrheic was counted as zero on both scales. Bleeding patterns and adverse effects were also recorded.

\section{Statistical analysis}

In this retrospective cohort analysis, the number of cases in the region during the study period determined the sample size. We did not perform a power analysis before the study as we planned to review all cases in our database. A power analysis was performed post hoc, however, to determine the statistical power of our results. The data were interpreted and verified by an independent observer. Continuous variables are expressed as the mean \pm standard derivation (SD) and binary variables are presented as frequencies and percentage. The comparison of pain intensity between the two study groups was performed using Student's t-test. A paired sample t-test was used to compare pain scores and endometrioma size for patients before (baseline) and after treatment (at 6 months) for within-group comparison. For binary variables, Fisher's exact test or Pearson's chi-squared test was applied when necessary. All statistical tests were two-sided. Values of $p<0.05$ were considered statistically significant. Data were analyzed using SPSS software version 20.0 (SPSS Science, Chicago, IL, USA).

\section{Results}

Of the 81 patients included in our study, 43 were in the dienogest group and 38 were in the dienogest plus ethinylestradiol group. None of the infertile women had any desire for pregnancy. Fifteen patients had one previous endometriosis surgery, and three patients had undergone more than one previous endometriosis surgery. The demographic characteristics of the patients are summarized in table I.

The pain scores and endometrioma size of the patients before treatment and at the 6-month follow-up are summarized in table II. In both groups (dienogest and dienogest plus ethinylestradiol), the pain scores related to chronic pelvic pain significantly decreased $36 \%$ and $49 \%$ respectively, $p<0.05)$ and dysmenorrhea significantly decreased (38\% and $44 \%$ respectively, $p<0.05$ ) at the 6 -month follow-up. A $28 \%$ decrease in the pain scores related to deep dyspareunia in the dienogest group was statistically significant at 6 months. Although pain scores in the dienogest plus ethinylestradiol group also decreased by $20 \%$, the difference did not reach significance $(p=0.09)$

There was no significant difference in pain scores between the two groups before treatment. Similarly, there was no significant difference between the two groups at the 6-month follow-up in chronic pelvic pain, dysmenorrhea, or deep dyspareunia pain scores.

Regarding endometrioma size, the mean size before treatment in the dienogest group decreased from $36.6 \pm 12.8 \mathrm{~mm}$ to $24.2 \pm 17.5 \mathrm{~mm}$ at the 6 -month follow-up. The $34 \%$ decrease in size was statistically significant $(p=0.02)$. The mean size before treatment in the dienogest plus ethinylestradiol group decreased from $38.7 \pm 13.2 \mathrm{~mm}$ to $27.5 \pm 19.1 \mathrm{~mm}$ at the 6 -month follow-up. The $29 \%$ decrease in size was statistically significant $(p=0.01)$. There was no significant difference in endometrioma size between the two groups at the 6-month follow-up (dienogest $24.2 \pm 17.5 \mathrm{~mm}$, dienogest plus ethinylestradiol $27.5 \pm 19.1 \mathrm{~mm}, p=0.42$ ).

The most frequent drug-related adverse effect in the dienogest group was spotting $(34.8 \%)$, which was significantly more common than that in the dienogest plus ethinylestradiol group $(15.7 \%, p=0.04)$. However, none of the patients discontinued treatment due to adverse effects. The most frequent drug-related adverse effect in the dienogest plus ethinylestradiol group was headache (21\%), which was not significantly higher than that in the dienogest group $(11.6 \%$, $p>0.05$, see table III). 
Table I: Baseline demographic characteristics of patients

\begin{tabular}{|c|c|c|c|}
\hline & $\begin{array}{l}\text { Dienogest } \\
(n=43)\end{array}$ & $\begin{array}{l}\text { Dienogest plus ethinylestradiol } \\
\qquad(n=38)\end{array}$ & $p$ \\
\hline Age, years & $32.7 \pm 6.07$ & $31.2 \pm 5.98$ & 0.36 \\
\hline BMI, kg/m² & $23.4 \pm 4.1$ & $24.3 \pm 3.2$ & 0.49 \\
\hline \multicolumn{4}{|l|}{ Parity } \\
\hline 0 & 15 & 12 & 0.38 \\
\hline $1 \leq$ & 28 & 26 & \\
\hline Abortion, n (\%) & $2(4.6 \%)$ & $3(7.8 \%)$ & 0.50 \\
\hline Infertility, n (\%)ף & $4(9.3 \%)$ & $6(15.7 \%)$ & 0.36 \\
\hline Age at menarche & $13.7 \pm 1.8$ & $13.5 \pm 1.8$ & 0.76 \\
\hline Cigarette smokers, n (\%) & $8(18.6 \%)$ & $6(15.7 \%)$ & 0.39 \\
\hline Previous endometriosis surgery & $11 / 43$ & $7 / 38$ & \\
\hline 1 & 9 & 6 & 0.28 \\
\hline$>1$ & 2 & 1 & \\
\hline Endometrioma size $(\mathrm{mm})^{¥}$ & $36.6 \pm 12.8$ & $38.7 \pm 13.2$ & 0.52 \\
\hline \multicolumn{4}{|l|}{ Laterality of endometrioma } \\
\hline Unilateral & 31 & 21 & 0.32 \\
\hline Bilateral & 12 & 17 & \\
\hline Chronic pelvic pain, n (\%) & $19(44.1 \%)$ & $22(57.8 \%)$ & 0.21 \\
\hline Dysmenorrhea, n (\%) & $32(74.4 \%)$ & $30(78.9 \%)$ & 0.12 \\
\hline Deep dyspareunia, n (\%) & $14(32.5 \%)$ & $18(47.3 \%)$ & 0.15 \\
\hline Chronic pelvic pain, years ${ }^{\&}$ & $4.2 \pm 3.6$ & $3.7 \pm 3.2$ & 0.48 \\
\hline Dysmenorrhea, years ${ }^{\&}$ & $4.9 \pm 4.6$ & $5.5 \pm 4.7$ & 0.42 \\
\hline Deep dyspareunia, years ${ }^{\&}$ & $2.5 \pm 1.6$ & $2.0 \pm 1.3$ & 0.21 \\
\hline
\end{tabular}

II None of the infertile women had any desire for pregnancy, \& Time since beginning pain, $n$ (\%); number of patients (percentage), BMI; Body mass index, $¥$ The largest diameter of the endometrioma on sonographic view was recorded as the size of the ovarian endometrioma, Data are presented as mean $\pm S D$ (standard deviation) unless otherwise noted

Table II: Pain scores and endometrioma size at baseline and 6 months

\begin{tabular}{|c|c|c|c|c|c|c|}
\hline Parameters $\pi$ & Baseline & 6 months & Mean decrease & $p^{\&}$ & $\% \S$ & $\begin{array}{c}\text { Required } \\
\text { sample size }\end{array}$ \\
\hline \multicolumn{7}{|c|}{ Chronic pelvic pain } \\
\hline D & $4.2 \pm 3.0$ & $2.6 \pm 1.9$ & $-\% 36$ & 0.02 & \multirow{3}{*}{27.7} & \multirow{3}{*}{ 181/181 } \\
\hline$D+E$ & $4.3 \pm 3.3$ & $2.2 \pm 1.7$ & $-\% 49$ & 0.00 & & \\
\hline$p^{\#}$ & .84 & .40 & & & & \\
\hline \multicolumn{7}{|c|}{ Dysmenorrhea } \\
\hline D & $5.7 \pm 2.6$ & $3.5 \pm 2.2$ & $-\% 38$ & 0.00 & \multirow{2}{*}{97.9} & \\
\hline$D+E$ & $5.5 \pm 2.9$ & $2.9 \pm 2.1$ & $-\% 44$ & 0.00 & & \\
\hline$p^{\#}$ & .78 & .29 & & & & \\
\hline \multicolumn{7}{|c|}{ Deep dyspareunia } \\
\hline D & $3.8 \pm 2.1$ & $2.7 \pm 1.7$ & $-\% 28$ & 0.03 & \multirow{2}{*}{92.7} & \\
\hline$D+E$ & $3.6 \pm 2.5$ & $2.9 \pm 2.3$ & $-\% 20$ & 0.09 & & \\
\hline$p^{\#}$ & .70 & .48 & & & & \\
\hline \multicolumn{7}{|c|}{ Endometrioma size } \\
\hline D & $36.6 \pm 12.8$ & $24.2 \pm 17.5$ & $-\% 34$ & 0.02 & \multirow{2}{*}{20.1} & \multirow{2}{*}{$251 / 251$} \\
\hline$D+E$ & $38.7 \pm 13.2$ & $27.5 \pm 19.1$ & $-\% 29$ & 0.03 & & \\
\hline$p^{\#}$ & .52 & .42 & & & & \\
\hline
\end{tabular}

" : A 0- to 10-point numerical rating scale (NRS), with "0" indicating no pain and "10" indicating the worst possible pain, was used, \&: $p$ value for within-group comparison (i.e. baseline vs. 6 months), §: Calculated post hoc power analysis, ¥: Required sample size for $80 \%$ power assuming a $5 \%$ significance level for each group, $D$, Dienogest-only; $D+E$, dienogest plus ethinylestradiol, \# : $p$ values for comparison between-groups (i.e. baseline, $D$ vs $D+E)$ 
Table III: Reported adverse effects at 6 months

\begin{tabular}{|c|c|c|c|}
\hline Parameters\& & $\begin{array}{l}\text { Dienogest } \\
\quad(n=43)\end{array}$ & $\begin{array}{l}\text { Dienogest plus } \\
\text { ethinylestradiol } \\
\quad(n=38)\end{array}$ & $p$ \\
\hline Spotting & $15(34.8 \%)$ & $6(15.7 \%)$ & 0.04 \\
\hline Weight gain & $6(13.9 \%)$ & $3(7.8 \%)$ & 0.30 \\
\hline Vasomotor symptoms & $9(20.9 \%)$ & $5(13.1 \%)$ & 0.26 \\
\hline Irritability & $10(23.2 \%)$ & $4(10.5 \%)$ & 0.11 \\
\hline Headache & $5(11.6 \%)$ & $8(21 \%)$ & 0.19 \\
\hline Nausea & $2(4.6 \%)$ & $3(7.8 \%)$ & 0.44 \\
\hline Discomfort from amenorrhea & $5(11.6 \%)$ & $7(18.4 \%)$ & 0.29 \\
\hline
\end{tabular}

Data are presented as number of patients (percentage) unless otherwise noted

\&No patients discontinued treatment due to adverse effects

\section{Discussion}

In this study, the effects and tolerability of dienogest and dienogest plus ethinylestradiol on endometriosis-related pain and endometrioma size were investigated. Pain scores in the dienogest and dienogest plus ethinylestradiol treatment groups were significantly lower for chronic pelvic pain and dysmenorrhea at the 6-month follow-up. Although dyspareunia was reduced by $21 \%$ in the dienogest plus ethinylestradiol group, this change was not significant, unlike in the dienogest group. Both groups also demonstrated significantly reduced endometrioma size.

Surgical treatment may be a mandatory option in the presence of subocclusive conditions (e.g. ureteral stenosis, bowel stenosis) and failure of medical therapies. The clinical management of endometriosis requires a clear understanding of the goals of surgery because surgical interventions can cause more ovarian damage than benign cysts, further decreasing ovarian reserve. Moreover, the recurrence rate for endometriosis after surgical excision may be as high as 50\% at 5 years because it is not possible to remove all viable endometriotic tissue. Repeat surgery for recurrent disease is associated with greater harm than the first surgery, as evaluated using antral follicle count and ovarian volume $(1,11)$. In addition, the complexity of the surgical procedure is often beyond simple excision of the endometriotic cyst or lesion and may require more extensive dissection (12). Many societies including the American College of Obstetricians and Gynecologists (ACOG) and the American Society for Reproductive Medicine (ASRM) endorse empiric therapy for endometriosis-related pain before a definitive surgical diagnosis $(1,13)$. In the current literature, there are few studies comparing oral progestin-only and combined oral contraceptive (COC). There is only one study directly comparing with the same progestin content (oral desogestrel vs. oral desogestrel plus ethinylestradiol) (14). In our study, two drugs with the same progestin quantity were compared (oral dienogest vs. oral dienogest plus ethinylestradiol), similar to Morotti et al. Although the ultimate effects of different synthetic progestins in the target tis- sue are similar, the drugs differ chemically and pharmacologically. The interaction of these progestins with other members of the steroid receptor family can cause unpredictable pharmacodynamic effects in the tissue (15).

There are numerous current medical treatments for the management of endometriosis symptoms. All of these treatments should be considered suppressive rather than curative. Hormonal management is by necessity long-term; the ideal regimen should be cost-effective, well tolerated, and without significant risk to the patient. Currently, intense debate continues in the literature about the first choice of medical therapies. COCs are low cost, well tolerated, and familiar to most women as contraceptives. Falcone et al. suggested that continuous COCs could be considered as the first-line treatment (16). In another review, Vercellini et al. suggested that COCs should be the first-line therapy for endometriosis pain, followed by progestin-only treatment in the case of a contraindication to estrogen. The authors emphasized that COCs, which could be used safely for many years, did not alter the serum lipid profile and did not decrease bone mineral content in contrast to progestins (2). In a similar study, COC treatment was found to be effective in all types of endometriosis-related pain (dysmenorrhea, pelvic pain, and dyspareunia) (3). In contrast, there was insufficient evidence to demonstrate that COCs are effective in managing pain-related symptoms compared with a placebo or other medical therapies in a Cochrane review (4). Moreover, considering potential progesterone resistance, there are concerns that the estrogen component in COCs may lead to progression of the disease by creating a localized hyper-estrogenic environment (17).

Combined oral contraceptive agents were reported as significantly reducing dysmenorrhea, pelvic pain, and dyspareunia from baseline in most studies $(3,14,18)$. In our study, similar to progestin-only therapy, a COC was effective on dyspareunia, but this difference was not significant at the 6-month follow-up. This result may be related to the small sample size or short-term follow-up in our study. Similarly, Casper et al. emphasized that COCs had no beneficial effects on dyspareu- 
nia (17). Progesterone receptor expression and progesterone sensitivity of these receptors are mediated by estrogens in eutopic endometrium. Estrogens also cause progesterone to become more potent by inhibiting its metabolism. In a previously reported study, the number of progesterone receptors in the estrogen-treated group was significantly increased compared to that in the untreated group in eutopic endometrium (19). In contrast, estrogen treatment in endometriotic tissues does not cause an increase in progesterone receptor expression and protein levels. Induction of progesterone receptor expression by estrogen in endometriosis is markedly blunted (20). Estrogen receptor alpha $(\mathrm{ER} \alpha)$ deficiency in endometriosis may be responsible for the failure of estrogen to induce progesterone receptor expression, thus contributing to secondary progesterone receptor deficiency and progesterone resistance in women with this disease. Consequently, the estrogen component of COCs may not have a positive impact on progesterone resistance in endometriosis lesions (21). There is only one study on how ER $\alpha$, which is responsible for progesterone resistance in the disease, is distributed in different types of endometriotic lesions. In this study, the ratio of ER $\alpha$ to estrogen receptor beta $(E R \beta)(E R \alpha / E R \beta)$ was significantly higher in red superficial peritoneal lesions compared with black peritoneal lesions and ovarian endometrioma (22). Considering the above-mentioned receptor-endometriotic lesion relationship, Vercellini et al. recommended the use of COCs in young patients with early peritoneal lesions in the lesion-based medical approach, and progestins in patients with deep dyspareunia arising from fibrotic nodules with no estrogen receptors (23).

The main limits of the current study are the small number of subjects in both groups, its retrospective nature, as well as the lack of surgical confirmation of endometriosis. Because patients were not selected randomly, selection bias may be a problem. However, patients were selected and assigned to study groups using rigorous exclusion and inclusion criteria to avoid bias. Due to the retrospective nature of the study, statistical power was affected by the number of patients available in the records. There was no significant difference between the therapeutic effects in the two groups at the end of 6 months of treatment with respect to chronic pelvic pain or endometrioma size, but the statistical power was low for both $(27.7 \%$ and $20.1 \%$, respectively). The required sample sizes to achieve an $80 \%$ power assuming an $\alpha$-type error of $5 \%$ for chronic pelvic pain and endometrioma size in each group were 181 and 251, respectively. In contrast, our study had sufficient power to compare dysmenorrhea and deep dyspareunia between the two groups (97.9\% and $92.7 \%$, respectively).

In conclusion, upon analysis of the data for the 6-months treatment period, estrogen-progestin and a progestin alone seem to be of similar efficacy for the temporary treatment of endometriosis-related pain. The dienogest plus ethinylestradiol combination was slightly less effective on deep dyspareunia but was well tolerated. Similarly, the two hormonal regi- mens posed no superiority over one another with regard to endometrioma size reduction. It may finally be stated that estrogen does not potentiate the therapeutic efficacy of dienogest in any of the parameters analyzed in this study. Further prospective controlled studies with larger sample sizes and longerterm follow-up should better define differences among the various hormonal regimens used to treat or alleviate symptoms of endometriosis.

Acknowledgments: The authors do not have any conflict of interest or financial support. This study was conducted in accordance with the Declaration of Helsinki and obtained the consent for using data.

Author contribution: TK: Data analysis and interpretation, wrote the manuscript, conceived the ideas of the study. HK: Data collection, conceived the ideas of the study. EO: Conceived the ideas of the study. MS: Data collection. EO: Revision of the manuscript.

\section{References}

1. Dunselman GA, Vermeulen N, Becker C, Calhaz-Jorge C, D'Hooghe T, De Bie B, et al. ESHRE guideline: management of women with endometriosis. Hum Reprod. 2014;29(3):400-12. DOI: 10.1093/humrep/det457.

2. Vercellini P, Buggio L, Berlanda N, Barbara G, Somigliana E, Bosari S. Estrogen-progestins and progestins for the management of endometriosis. Fertil Steril. 2016;106(7):1552-71. DOI: 10.1016/j.fertnstert.2016.10. 022.

3. Jensen JT, Schlaff W, Gordon K. Use of combined hormonal contraceptives for the treatment of endometriosisrelated pain: a systematic review of the evidence. Fertil Steril. 2018;110(1):137-52. DOI: 10.1016/j.fertnstert. 2018.03.012.

4. Brown J, Crawford TJ, Datta S, Prentice A. Oral contraceptives for pain associated with endometriosis. Cochrane Database Syst Rev. 2018;5:CD001019. DOI: 10.1002/ 14651858.CD001019.pub2.

5. Roth K, Zahradnik HP, Schäfer WR. Effects of different progestins on prostaglandin biosynthesis in human endometrial explants. Contraception. 2019;99(1):61-6. DOI: 10.1016/j.contraception.2018.08.001.

6. Andres Mde P, Lopes LA, Baracat EC, Podgaec S. Dienogest in the treatment of endometriosis: systematic review. Arch Gynecol Obstet. 2015;292(3):523-9. DOI: 10.1007/s00404-015-3681-6.

7. McCormack PL. Dienogest: a review of its use in the treatment of endometriosis. Drugs. 2010;70(16):2073-88. DOI: $10.2165 / 11206320-000000000-00000$.

8. Timmerman D, Ameye L, Fischerova D, Epstein E, Melis GB, Guerriero S, et al. Simple ultrasound rules to distinguish between benign and malignant adnexal masses before surgery: prospective validation by IOTA group. BMJ. 2010;341:c6839. DOI: 10.1136/bmj.c6839. 
9. Seyhan A, Urman B, Turkgeldi E, Ata B. Do endometriomas grow during ovarian stimulation for assisted reproduction? A three-dimensional volume analysis before and after ovarian stimulation. Reprod Biomed Online. 2018;36(2):239-44. DOI: 10.1016/j.rbmo.2017.10.108.

10. Bourdel N, Alves J, Pickering G, Ramilo I, Roman H, Canis M. Systematic review of endometriosis pain assessment: how to choose a scale? Hum Reprod Update. 2015;21(1):136-52. DOI: 10.1093/humupd/dmu046.

11. Stochino-Loi E, Darwish B, Mircea O, Touleimat S, Millochau JC, Abo C, et al. Does preoperative antimullerian hormone level influence postoperative pregnancy rate in women undergoing surgery for severe endometriosis? Fertil Steril. 2017;107(3):707-13.e3. DOI: 10.1016/j. fertnstert.2016.12.013.

12. Chapron C, Pietin-Vialle C, Borghese B, Davy C, Foulot $\mathrm{H}$, Chopin N. Associated ovarian endometrioma is a marker for greater severity of deeply infiltrating endometriosis. Fertil Steril. 2009;92(2):453-7. DOI: 10. 1016/j.fertnstert.2008.06.003.

13. ACOG Committee on Practice Bulletins--Gynecology. Practice bulletin no. 114: management of endometriosis. Obstet Gynecol. 2010;116(1):223-36. DOI: 10.1097/ AOG.0b013e3181e8b073.

14. Morotti M, Remorgida V, Venturini PL, Ferrero S. Progestogen-only contraceptive pill compared with combined oral contraceptive in the treatment of pain symptoms caused by endometriosis in patients with migraine without aura. Eur J Obstet Gynecol Reprod Biol. 2014; 179:63-8. DOI: 10.1016/j.ejogrb.2014.05.016.

15. Bastianelli C, Farris M, Rosato E, Brosens I, Benagiano G. Pharmacodynamics of combined estrogen-progestin oral contraceptives 3. Inhibition of ovulation. Expert Rev Clin Pharmacol. 2018;11(11):1085-98. DOI: 10.1080/ 17512433.2018.1536544.

16. Falcone T, Flyckt R. Clinical Management of
Endometriosis. Obstet Gynecol. 2018;131(3):557-71. DOI: $10.1097 /$ AOG.0000000000002469.

17. Casper RF. Progestin-only pills may be a better first-line treatment for endometriosis than combined estrogen-progestin contraceptive pills. Fertil Steril. 2017;107(3):533-6. DOI: 10.1016/j.fertnstert.2017.01.003.

18. Caruso S, Iraci M, Cianci S, Fava V, Casella E, Cianci A. Comparative, open-label prospective study on the quality of life and sexual function of women affected by endometriosis-associated pelvic pain on $2 \mathrm{mg}$ dienogest $/ 30$ $\mu \mathrm{g}$ ethinyl estradiol continuous or 21/7 regimen oral contraceptive. J Endocrinol Invest. 2016;39(8):923-31. DOI: 10.1007/s40618-016-0460-6.

19. Bryś M, Szyłło K, Romanowicz-Makowska H, Dobrowolski Z, Masłowska I, Krajewska W. Expression of estrogen and progesterone receptor genes in endometrium, myometrium and vagina of postmenopausal women treated with estriol. Sao Paulo Med J. 2009;127 (3):128-33. DOI: 10.1590/S1516-3180200900 0300004

20. Bulun SE, Cheng YH, Yin P, Imir G, Utsunomiya H, Attar $\mathrm{E}$, et al. Progesterone resistance in endometriosis: link to failure to metabolize estradiol. Mol Cell Endocrinol. 2006;248(1-2):94-103. DOI: 10.1016/j.mce.2005.11.041.

21. Bulun SE, Monsavais D, Pavone ME, Dyson M, Xue Q, Attar E, et al. Role of estrogen receptor- $\beta$ in endometriosis. Semin Reprod Med. 2012;30(1):39-45. DOI: 10.1055/ s-0031 1299596.

22. Matsuzaki S, Murakami T, Uehara S, Canis M, Sasano H, Okamura K. Expression of estrogen receptor alpha and beta in peritoneal and ovarian endometriosis. Fertil Steril. 2001;75(6):1198-1205. DOI: 10.1016/S0015-0282(01) 01783-6.

23. Vercellini P, Buggio L, Frattaruolo MP, Borghi A, Dridi D, Somigliana E. Medical treatment of endometriosis-related pain. Best Pract Res Clin Obstet Gynaecol. 2018;51:68-91. DOI: 10.1016/j.bpobgyn.2018.01.015. 